\title{
Methodology of Research into Social Aspects of Leprosy Control
}

\author{
CORLIEN M. VARKEVISSER \\ Department of Social Research, \\ Royal Tropical Institute, Amsterdam
}

\begin{abstract}
Given the goal of optimal dapsone intake, social scientific research needs to take into consideration both the socio-cultural and socio-medical settings in which dapsone is available.

Various techniques can help reveal what factors determine prompt self-reporting and regular clinic attendance, and what factors retard them. In our project (Western Province, Kenya and Mwanza Region, Tanzania 1974-76) we combined a factor analysis of data on patient registration cards (limited in value because of the low quality of the data) with in-depth interviewing (patients, relatives, neighbours, false-alarmists, community leaders, traditional doctors). With a set of "test" statements, we measured prevailing community attitudes towards leprosy patients and then compared the results with our observations. At the same time we interviewed health personnel intensively, and observed patient-staff interactions.

In-depth research is able to generate valuable suggestions for strengthening the leprosy services available, for training and retraining health personnel, and for educating patients and communities about leprosy control essentials.
\end{abstract}

Before selecting appropriate research methods for a particular investigation, the problem to be studied, and the purpose of the study must be carefully defined. If this problem is of a practical nature--deficient case-finding and case-holding in leprosy control - and if the purpose of the enterprise is to propose possible solutions, then research must concentrate on gathering data immediately relevant to decision making. It should also produce results as quickly as possible, for those who take the decisions--doctors, in our case-will, presumably, be eager to implement the results. Costs must form another consideration in the choice of research procedures. Where a country's resources are barely sufficient to cover the basic needs of its inhabitants, it is imperative to take a hard look at the kinds of results yielded by some of the expensive research techniques developed in Western societies. To what extent are these techniques appropriate in a non-Western situation, and do the benefits justify the costs? Never, however, should restrictions in available time and money seduce us into coming up with results that are superficial, and thus misleading. To put it briefly: the research must be relevant, reliable, quick, and cheap. 
I should like to elaborate on these points by critically evaluating research we carried out between 1974 and 1976 in two leprosy control schemes in East Af rica, our team consisting of five medical anthropologists and sociologists. The problem seemed clear: both in the Mwanza Regional Leprosy Control Scheme in Mwanza Region, Tanzania, and in the West Kenya Leprosy Control Project in Western Province, Kenya, case-finding and case-holding achievements were disappointing. Although between 65 and $80 \%$ of the estimated case-load in Mwanza Region and Western Province had been traced, when we began our research only about $30 \%$ of the patients registered were "under control", i.e., under regular (out-patient) treatment or officially declared cured. That potentially infectious cases appeared slightly more regular in attendance offered only minor consolation, when almost half of all the registered leprosy patients were lost sight of before being officially discharged. Could we, the doctors asked, help find out why most patients came forward for treatment in a relatively late stage of their disease? why so many "defaulted", and why others never showed up at all? Could we assist in formulating recommendations for the improvement of case-finding and case-holding activities, and could we suggest ways to implement these recommendations?

To start with, we redefined the problem. We identified case-finding and caseholding difficulties as communication problems involving two parties: the medical staff, with services to off er, and patients and other community members, with a need for these services. It was clear that both parties required study if we were to understand weak points in their mutual understanding. Such study takes time. Before a social scientist can make a useful contribution, he must both learn the ins and outs of the medical organization in question, and the ins and outs of the society within which the medical organization operates. Our research in leprosy control problems in Mwanza Region and Western Province occupied us, collectively, for almost two years. This first experience in East Af rica, however, has enabled us to come to grips with essential issues more efficiently in places where we have worked since (e.g. Nigeria, and Botswana's National Tuberculosis Programme).

When selecting our research methods we could choose between two different approaches. One is the so-called quantitative or survey approach, developed by Western sociologists. Among a carefully selected sample of the population under study-which may include hundreds of informants - sociologists systematically gather information which they assume will give them insight into the problems they are investigating. To this end they devise strictly structured questionnaires. Answers are usually precoded to facilitate data processing by computer. The computer analyses which variables influence each other and to what degree. Thus, to illustrate from our own field of study, we can find out to what extent a favourable or unf avourable attendance of leprosy patients is related to their sex, age, type of leprosy, economic situation, or level of education. By means of a factor analysis, the computer is, moreover, able to reveal the comparative importance of the different variables related to patients' attendance. A patient's economic status, for example, may prove more decisive for compliance with treatment prescriptions than his type of leprosy or degree of deformities. 
The other research approach stems from anthropology. It is sometimes labelled as the qualitative or "understanding" approach. The main research method has become known under the term "participant observation". By living among the people he is investigating, an anthropologist becomes nolens volens involved in the life of the community, even though his participation is as a rule largely passive. By keeping his eyes and ears wide open - and instructing his one or two well-trained research assistants to do the same-he will gather some extremely valuable information which he could never have obtained by means of questioning only. Even so, nowadays most anthropologists will, in addition, also question representative samples of informants on specific topics of interest. It would otherwise be impossible to generalize findings pertaining to the entire research population. Still, the anthropologist's technique of questioning differs significantly from the sociologist's. It is less fixed, and in leaving space for discussion with the informant, it creates opportunities for relevant information to merge spontaneously in the course of such discussions. . . the more, the better. An anthropologist, in trying to understand the problem he is investigating as thoroughly as possible, will prefer to go back to the same informant four or five times, rather than end up with a collection of superficial impressions by talking only once to five times as many informants. Consequently he will work with a limited number of informants, and usually in a restricted area. No matter how thought-provoking his conclusions may be, however, further research must demonstrate whether and to what extent they are applicable in other areas as well. The anthropologist's qualitative approach, therefore, has its limitations. But so does the survey approach. Sociologists themselves have expressed their doubts about the value of mass surveys, especially in developing countries, where several studies have clearly shown how difficult it is to obtain reliable answers by means of such data gathering (Pausewang, 1973).

Since both methods have their merits and weaknesses, a combination seems to offer better prospects for fruitful research. This is what we attempted in the Mwanza and West Kenya leprosy control schemes. In both areas we began with a quantitative analysis of patients' medical history cards and attendance records, a sample of 1760 cards in Mwanza Region, and of 1000 cards in Western Province. By feeding coded data to a computer, we expected to obtain detailed information about the composition of the patient population with regard to age, sex, type of leprosy, development of the disease, attendance history, geographical distribution, which would enable us to define a sample of patients for further in-depth interviewing. Also, we hoped to identify some variables affecting attendance behaviour. The preparations in the field for the computer analysis took five months. The analysis itself ( 2 months) took place in Holland. Within five months, a report with results was available. Our remaining year and a half in the field was spent interviewing and observing in two areas in Western Province inhabited by the Luhya, and in one larger area in Mwanza Region where the Sukuma live. The Sukuma like the Luhya, are a Bantu speaking tribe. We spoke with some 200 patients, with their relatives, neighbours, other members of the community (both prominent and less prominent), with traditional doctors, and with some 115 representatives of the leprosy and general health services scattered throughout Western Province and Mwanza Region. 
The quantitative analysis which we undertook proved costly, both in terms of time and money. And, no matter how technologically advanced a computer may be, if data are incomplete and unreliable, computer calculations can be of only limited value. For our factor analysis of variables related to attendance, patient cards and attendance registers provided data which varied considerably in quality. They contained - or, were supposed to contain - a number of medical variables, which we assumed would influence a patient's motivation to come for treatment. Among them: activity of the disease, reactions, number and visibility of lesions, degree of deformity. Further, in providing data on the organization and quality of the services, they contained a number of medical-sociological variables such as: training of fieldworker, amount of supervision (the last date on which any assessment of the patient's clinical activity had been made proved an effective clue), the number of home-visits paid, home-clinic distance. These cards, however, contained hardly any socio-cultural variables. Only the patient's age and sex were recorded; there was nothing recorded about level of education or the patient's socio-economic status.

Not only were a number of crucial variables lacking, especially in the sociocultural category, but also, data that should have been filled in were all too frequently missing, or unreliable. Attendance records sometimes seemed to have led a life of their own, irrespective of a fieldworker's presence or absence on clinic days.

The principal reason, however, for the limited utility of our attempts at finding out by means of a factor analysis which variables were related to patients' attendance was that there existed such fluctuations in the actual functioning of the leprosy services themselves. When services are provided irregularly, or even discontinued at certain clinics, complicated calculations in order to reveal which patients default and why, become meaningless. At least a rudimentary service must exist, continuously, throughout the entire area (tablets must always be available at the appointed time and place), before it makes sense to undertake such an analysis.

Af ter these qualif ying remarks it seems legitimate to ask: did we gain anything at all from the analysis? Fortunately, we did.

(1) We did not get a $100 \%$ accurate, but still the best possible picture of the composition of the patient population. From this we could select our sample of patients and ex-patients for in-depth interviews.

(2) Especially in Mwanza, where patient data were more complete and reliable than in Western Province, cross-tabulations between patients' attendance behaviour and available variables gave at least some insight into the identity of those who come regularly for treatment, and those who don't, e.g. deformed patients, who reported themselves with leprosy when middle-aged, and who live not further than five miles from their leprosy clinic, are predictably better attenders than those without deformities, who contract leprosy in their youth or old age, are found in a school or mass survey and/or are living further than five miles away from a treatment point.

(3) Cohort analysis of patients' disease- and attendance-history, per year of registration (the only way to thoroughly evaluate the progress made by a leprosy scheme and to compare the achievements of different schemes), became comparatively easy. 
(4) Generalization about individual attendance patterns became feasible. We only did this in Mwanza, where attendance data were relatively complete. We found that by far most patients "default" within two years after being registered. Once a patient has established a stable pattern of visiting clinics, he appears to carry on. This finding proves how urgent it is to devote special attention to new patients, offering them relevant health-education, and visiting them after any early absence.

(5) The results of the computer analysis evoked more questions than it solved, thus providing a challenge for the next phase of in-depth interviewing. It was, for instance, clear that socio-cultural factors could not explain why in a culturally homogeneous, predominantly rural area, the percentage of regularly attending patients varied from 20 to $90 \%$ within a radius of $100 \mathrm{~km}$. We would have to look as deeply into the organization of the service and the motivation of leprosy workers to do their jobs, as into the socio-cultural background of patients and their motivation to come for treatment. Difference in quality of the service became an important criterion for the choice of the areas in which we conducted our interviews. In Western Province we selected the Wanga locations because part of the services appeared to function very well, the Busuku locations because they functioned rather poorly. As fieldwork progressed we discovered that the Wanga's provided an even more interesting research situation than expected: its two fieldworkers differed significantly in dedication to their work. Under such circumstances it is as interesting to find out which patients disappear from treatment and why, even when the service is reasonable or good, as it is to know which patients will struggle to get treatment, even if the service functions poorly.

The question remains whether a computer analysis is the best way to obtain these positive results in countries where computers are scarce and have long waiting lists of clients. In our eyes the answer must be NO. Hertroys' (1974) analysis of every tenth patient registration card in Mwanza, and our own analysis (Varkevisser, 1977; Paape and Varkevisser, 1978) of some 2000 TB patient cards in Botswana, by hand, with the assistance of a small calculator only, produced simple but relevant information many times more cheaply, and more quickly.

Far more rewarding and revealing than our quantitative analysis of medical records - both for ourselves and, we feel, for the doctors-were our in-depth interviews. The technique we used when speaking with patients and ex-patients can be described as a topic-wise, loosely structured interview. Together we developed a schedule of items we wanted to cover, rather than a strict questionnaire. Generally, we would pose some leading questions: "How did your disease begin?" and "How do you feel now?" ... then letting the patient determine the further course of topics. Within limits, of course. In the evenings we organized notes, making sure that we had obtained all the information we wanted. Wishing to understand the full process each patient had gone through, from the moment of first noticing a possible leprosy symptom down to the present, we time and again returned to the same informant, encouraging him or her to talk about their lives. In addition, we tried our best to speak with many 
satellite informants: the spouses, relatives, and neighbours of a patient. This was not only to verify what we had heard from the patients themselves, but also to enrich our picture of reality. How had the patients, how had their neighbours and relatives reacted to the disease? Of particular importance to this stage of our research was an evaluation of the role of treatment, both traditional and modern, in patient-community relations.

Our team intentionally discarded the idea of working with fixed questionnaires such as used, for example, in KAP (Knowledge, Attitude, Perception) studies. One reason for this decision was that patients, when talking about their lives, are reserved. They are hesitant to reveal painf ul encounters with relatives or community members, reluctant to confess how long it took them to report for modern treatment. Often, it wasn't until during a later interview that we could discover how earlier on we had been deliberately deceived. Our one guiding rule was never to accept any statement at its face value. We had to develop patience, until successive interviews with patients, relatives, and fellow villagers generated a consistent picture. A fixed questionnaire would no doubt have yielded results ... quite specific ones, quite readable and, we are convinced, quite misleading and superficial!

The stress of such probing, in-depth research on the one carrying it out is considerable. There is always that gap between what you want to know and what you get to know. It is a gap that can only be bridged slowly and gradually, if ever. Often the most valuable information, the real "eye openers" will come unexpectedly. And yet, the researcher must at all costs avoid being suggestive, especially where such relatively free interviews do entail the danger of his own personality introducing a strongly subjective element. Close cooperation with fellow researchers, however, can help correct this.

Although we judged strictly structured questionnaires unsuitable for teaching us much about the reality of interaction between patients, community, and health staff personnel, they did prove useful in bringing to light the generally accepted "code of behaviour" towards leprosy patients. In an attempt to specify the areas of social interaction where the stigma surrounding leprosy was relatively intense or weak, we drew up a set of statements concerning both actual and wished for behaviour on the part of leprosy patients. For example: "A leprosy patient cannot marry", and "A leprosy patient should not be allowed to marry". We then asked informants if they agreed, disagreed, or agreed in part with the statements. Then we invited the informant to comment. This aspect of our study showed us that there was a widespread discrepancy between, on the one hand, fear of "the leprosy patient" as a stereotype, with the accompanying desire to impose restrictions on his behaviour and, on the other hand, the actual tolerance we observed towards specific patients. In their dealings with a particular relative or neighbour who had leprosy, people would largely maintain ordinary forms of behaviour ... if, that is, the patient was receiving treatment, and/or his disease "cooled down".

For interviews with health staff, we used a combination of topic-wise, relatively free interviews, and a number of fixed questions, albeit open-ended. We employed free interviews; we wanted staff's opinion on bottlenecks in the service, and on possible remedies; we -were more pointed when we wished to 
learn about the procedures followed in their treatment of patients, and to test their knowledge about leprosy.

Finally, observation, followed by discussions with research assistants about what we had seen, formed an invaluable, complementary research method. We deliberately frequented social gatherings (beer clubs, markets, celebrations), in order to observe interaction between patients and community members, and asked our research assistants to do the same, often by themselves. They, with or without us, systematically attended clinic days in our research area to observe over time the interaction between health staff and patients. This way we could evaluate the leprosy services from three angles: from what health staff had told us, from what patients had told us, and from our own observations.

By way of summary, one could say that, although the various methods we used all had drawbacks as well as merits, in combination they worked well. Those team members who have remained involved in this type of investigation feel, however, that research is most fruitful when it is clear from the beginning that researchers will share in the responsibility for implementing results. On this basis we are now working in Botswana, hoping to soon start in Indonesia as well. Our practical contribution principally concerns the field of health education, the training of staff, and (re)organization of treatment procedures at base level.

\section{Acknowledgements}

Team members were Iman Bijleveld, Titus J. K. Gateere, Carla I. Risseeuw, Jan G. J. M. Ruyssenaars and Corlien M. Varkevisser. The study was made possible by grants from the Dutch Directorate for Technical Assistance, the Foundation for Scientific Research in the Tropics, the Netherlands Leprosy Relief Association and the Royal Tropical Institute.

Research reports are available at the Royal Tropical Institute, Mauritskade 63, Amsterdam, Holland

\section{Ref erences}

Hertroys, A. R. (1974). A study of some factors affecting attendance of patients in leprosy control scheme. Int. J. Lepr. 42, 419.

Paape, M. and Varkevisser, C. M. (1978). Evaluation of attendance behaviour of TB patients who were registered at Athlone Hospital, Lobatse and at SDA Hospital, Kanye, during 1976 and 1977, and of patients' intake of medicines. Preliminary Report, Ministry of Health, Botswana, 1978.

Pausewang, S. (1973). Methods and Concepts of Social Research in the Rural Developing Society. Weltforum, Munchen, 1973.

Varkevisser, C. M. (1977). TB control in Botswana; problem identification. Report to the Royal Tropical Institute, Amsterdam, 1977. 